\title{
Amphetamines in the treatment of adult attention-deficit/ hyperactivity disorder (ADHD) and cocaine use disorder (CUD): The role of pharmacists
}

\author{
Athena Milios, B.Sc., M.Sc.' \\ I. College of Pharmacy, Dalhousie University
}

\begin{abstract}
Attention-Deficit/Hyperactivity Disorder (ADHD) is a neurodevelopmental disorder characterized by inattentiveness, hyperactivity, and impulsivity. This review paper outlines the role that pharmacists can play in monitoring amphetamine use, to reduce the possibility of medication abuse by those with ADHD and/or Cocaine Use Disorder (CUD). Because individuals with ADHD also struggle with impulsivity, they are more likely to abuse substances, particularly illegal stimulants (such as cocaine), in an effort to self-medicate. This article also reviews the pharmacokinetics of amphetamine derivatives as well as the evidence for their use to manage ADHD and CUD. Neuropharmacologically, the proposed mechanism of action of amphetamines in the treatment of CUD is also detailed. Finally, the implications of these findings for pharmacy practice are discussed. The primary findings and principal conclusions are that amphetamines have been found to improve both ADHD and CUD symptomatology, primarily through increasing DA release from nerve terminals in the central nervous system, as well as increasing the release of NE and serotonin. Pharmacists can play an important role in monitoring use of these medications by working in collaboration with family physicians and psychiatrists to ensure that ADHD/CUD patients are taking their amphetamines as prescribed.
\end{abstract}

\section{Background on ADHD and CUD}

A ttention-deficit/hyperactivity disorder (ADHD) is a neurodevelopmental disorder characterized by inattentiveness, hyperactivity, and impulsivity ${ }^{1}$. The symptoms of ADHD include restlessness, the urge to be "going" nonstop, impatience, talking very fast, being forgetful, losing things easily, an inability to focus, and difficulties sustaining attention on important daily life tasks ${ }^{1}$. Roughly $80 \%$ of children with ADHD will continue to meet diagnostic criteria for the disorder into their adolescent years and approximately $60 \%$ will maintain the core symptoms of the disorder into their adult years ${ }^{2}$. Amphetamine derivatives are used in ADHD because they help with maintaining focus by increasing levels of dopamine and norepinephrine in brain pathways involved in attention, reinforcement, and reward; however, this means they also have a high potential for dependence and abuse.

Cocaine Use Disorder (CUD) is defined as a pattern of cocaine use that leads to clinically significant impairment or distress evidenced by at least two of the following symptoms in a 12-month period: (1) cocaine is used in larger amounts or over a longer period than intended, (2) the patient displays a desire or unsuccessful attempts to control/cut down their use, (3) the patient spends significant amounts of time trying to acquire cocaine, use cocaine, or recover from its effects, (4) the patient craves cocaine, (5) the patient is significantly impaired in the domains of work, school, and/or home due to their cocaine use, (6) the patient continues using cocaine despite persistent/recurring interpersonal issues due to their cocaine use, (7) the patient reduces/stops important social/occupational/ recreational activities due to cocaine use, (8) the patient uses cocaine in situations where they are in physical danger, (9) the patient continues using cocaine despite knowing that they have a physical/psychological problem caused or exacerbated by cocaine use, (10) the patient displays tolerance to cocaine, and (11) the patient displays symptoms characteristic of stimulant withdrawal when they are not using cocaine ${ }^{1}$.

Pharmacists can play a central role in monitoring amphetamine use to reduce the possibility of it being abused, both in individuals with ADHD as well as in individuals with comorbid ADHD and CUD. The issue of proper stimulant use is very relevant in modern society because there are great personal and societal costs associated with stimulants, such as adverse health effects, premature deaths, and higher crime rates in certain communities ${ }^{3}$.

Because patients with ADHD struggle with impulsivity, they are statistically more likely to abuse substances, particularly illegal stimulants (of which co- 
caine is the most common), but also prescription stimulants (of which the amphetamine derivatives are the most common), in an effort to self-medicate ${ }^{4,5}$. Given that amphetamines are quite effective in the treatment of $\mathrm{ADHD}^{6}$, they have also been investigated for the treatment of CUD, which is another disorder linked to deficient dopamine transmission, like ADHD. Comorbid ADHD and CUD worsens the prognosis, and these patients have poorer long-term outcomes in many life domains ${ }^{5}$.

Patients who misuse cocaine may be self-medicating latent/undiagnosed ADHD, therefore assessment of these patients for ADHD is very important - in patients with comorbid ADHD and CUD, treating their ADHD could dramatically improve their psychosocial functioning and help to address the underlying factors contributing to their cocaine abuse ${ }^{5}$. Individuals who have higher baseline ADHD symptomatology have been found to be at a much higher risk for misusing cocaine and other illegal stimulants 5 . This epidemiological association underscores the importance of diagnosis and formal testing in all patients who abuse stimulants, whether they are stimulants that are available by prescription or illegal stimulants such as cocaine.

This review paper will examine the evidence for the potential role of interdisciplinary collaboration between pharmacy, medicine, and other healthcare disciplines in treating patients with comorbid ADHD and CUD. Furthermore, an association between ADHD and CUD has been established in the literature and has a neurophysiological basis, which will also be discussed.

\section{Pharmacokinetics}

When ingested via the oral route, amphetamines have a bioavailability of roughly $75 \% \%^{7}$. The immediate-release (IR) formulations require one to three hours to reach their peak effect; their duration of action is roughly four to six hours ${ }^{8}$. Extended-release (ER) or sustained-release (SR) formulations of amphetamines typically last longer in the body, with a duration of action of approximately ten hours ${ }^{8}$. Because of their pharmacokinetic properties (both the longer duration of action and the slower/lower peak plasma level), ER and SR formulations also have a lower potential for abuse ${ }^{8}$.

\section{Evidence for Amphetamines in CUD}

Mooney and colleagues (2009) found that the use of amphetamine analogues can lead to significant reductions in cocaine use as well as cravings. Chronic cocaine use has been found to deplete dopaminergic, serotonergic, and noradrenergic pathways in the brain, leading to psychiatric symptoms that include increased impulsivity, depression, and anhedonia' ${ }^{9}$ Amphet- amine derivatives can help to target these symptoms and are associated with reductions in cocaine use as well as cocaine cravings ${ }^{9,10,11}$. However, it is only the SR or ER amphetamine derivatives that have been found to be effective for treating CUD, not the IR formulations, which need to be taken multiple times a day (due to their shorter duration of action) and are more likely to be abused ${ }^{8,9}$. Levin and colleagues (2015) conducted a randomized clinical trial showing that ER amphetamine salts (in addition to Cognitive Behavioral Therapy) are effective in the treatment of individuals with comorbid ADHD and CUD. The ER amphetamine salts were found to improve ADHD symptoms in addition to reducing cocaine use, further supporting the theory that individuals with ADHD are more likely to use cocaine as a form of self-medication to address their underlying ADHD symptoms ${ }^{5}$.

While the role for amphetamine derivatives has been well established in the treatment of patients with comorbid ADHD and CUD, adding psychotherapy (e.g., Cognitive Behavioral Therapy) to amphetamine-based pharmacotherapy could also confer additional clinical benefit to help patients achieve and maintain cocaine abstinence, although more research is needed in this domain ${ }^{10}$. Patients with co-occurring ADHD and CUD that achieve cocaine abstinence at the beginning of treatment while treated with amphetamines (to address their core ADHD symptoms) appear to have better outcomes in achieving/maintaining CUD remission; this is likely due to an improvement in ADHD symptoms ${ }^{11}$. These findings support the self-medication theory of stimulant abuse in patients with ADHD. Several well-designed large-scale studies examining amphetamine derivatives in the treatment of ADHD and comorbid CUD have shown promising results ${ }^{4,10,11}$.

\section{Mechanism of Action}

Amphetamine's mechanism of action is on the monoamine neurotransmitters dopamine (DA), norepinephrine (NE), and serotonin ${ }^{6}$. It is considered an indirect-acting sympathomimetic and is associated with increases in blood pressure, heart rate, body temperature, and cardiac output, in addition to reducing appetite $^{6}$. Amphetamines are weak reuptake inhibitors of DA and serotonin, and moderate reuptake inhibitors of $\mathrm{NE}^{6}$. Amphetamines enter NE neurons via the norepinephrine transporter (NET) and are taken into storage vesicles by the vesicular monoamine transporter (VMAT). By moving into NE storage vesicles, amphetamines displace NE into the cytoplasm of presynaptic neurons. Subsequently, there is movement of NE into the synaptic cleft. No action potential is needed for this release of NE into the synapse; the movement is triggered by amphetamine molecules moving NE out 
of their storage vesicles in the presynaptic neuron. The $\mathrm{NE}$ in the synapse then stimulates adrenergic receptors on postsynaptic neurons.

In regard to DA, amphetamines block the ability of the dopamine transporter (DAT) to clear DA from the synapse, leading to increased synaptic DA concentrations. Amphetamines also bind to VMAT, leading to DA transfer reversing so that higher concentrations of DA end up moving into the synapse from storage vesicles $^{6}$.

Amphetamines block NET and SERT (the serotonin transporter) as well, leading to higher concentrations of NE and serotonin in the synapse in addition to increasing synaptic DA levels ${ }^{6}$. Regular cocaine use depletes dopaminergic and noradrenergic systems in the brain and can have lasting effects on impulse control. Since amphetamines target these systems in the brain, they can improve some of the psychiatric symptoms experienced by CUD patients.

In terms of the brain regions they affect, amphetamines lead to increases in DA and NE in the prefrontal cortex and the striatum. Amphetamines are highly reinforcing, primarily due to increased DA release in the synapses within the mesolimbic (reward) pathway of the brain, which includes the nucleus accumbens (part of the ventral striatum $)^{5}$. The nucleus accumbens is a central part of the brain's reward circuit and is implicated in both ADHD and CUD.

\section{Implications for Pharmacy Practice}

The pharmacy model of care that could be implemented when pharmacists are monitoring patients receiving treatment for comorbid ADHD and CUD has parallels to the role that pharmacists currently play in methadone maintenance treatment. Firstly, it is important that pharmacists establish a strong therapeutic pharmacist-patient relationship with ADHD/CUD patients, based on a foundation of mutual respect, empathy, compassion, and understanding ${ }^{12}$. Pharmacists should treat substance use disorder patients as they would all other patients.

In community pharmacy practice, pharmacists can play an important role in monitoring amphetamine use by working in close collaboration with family physicians and psychiatrists to ensure that ADHD/CUD patients are taking their amphetamines as prescribed. As part of this collaboration, there should also be regular communication between pharmacists and physicians so that there is safe, seamless, uninterrupted treatment for ADHD/CUD patients, especially when they are transitioning from inpatient to outpatient institutions and when they are switching pharmacies ${ }^{12}$. Thorough documentation is also important to ensure that pharmacists are able to collaborate effectively with other healthcare providers who are treating their ADHD/CUD patients ${ }^{12}$. Pharmacists can also educate patients (e.g., using behavioral approaches) ${ }^{3}$ regarding the adverse effects of stimulants, in order to reinforce the benefits of using stimulants as prescribed and the consequences of stimulant abuse. Pharmacists should also ensure that they keep up to date on the clinical knowledge and research surrounding both ADHD and CUD, as well as any relevant clinical practice guidelines that are published when they are treating this specific patient population ${ }^{12}$. Lastly, pharmacists must keep a close eye out for early renewal requests for amphetamines in ADHD/CUD patients as well as attempts by these patients to get their amphetamines prescribed by multiple physicians, as these could be subtle but important signs that amphetamines are being misused.

Because patients generally have good relationships with pharmacists, pharmacists are among the most readily accessible and highly trained healthcare professionals, and the fact pharmacists work long hours (which include weekends/holidays), pharmacists are in an excellent position to ensure that patients with comorbid ADHD/CUD are taking their prescription stimulants properly. Management of ADHD and CUD must be targeted using a multimodal approach, which includes medications, education, and behavioral interventions. Pharmacists have an integral role to play in this targeted multimodal approach because they are a key player in complete and comprehensive therapeutic interventions for any given patient. Pharmacists play a vital role in continuation of patient care and in improving long term outcomes for patients suffering with these chronic and often difficult-to-treat disorders.

\section{Conclusions}

In patients with comorbid ADHD and CUD, these neuropsychologically linked disorders can be addressed together and in a safe manner. Pharmacists can have an important role in overseeing treatment adherence and minimizing the risk of diversion (of stimulant medications from those with ADHD to others) or abuse of amphetamine derivatives ${ }^{5}$.

This paper focused on reviewing the literature linking ADHD and CUD, their treatment individually and comorbidly, and the role that pharmacists can play in monitoring the treatment of these patients. This is an important topic that is generally poorly understood and historically understudied. In typical clinical practice, clinicians avoid prescribing stimulants for patients with cocaine abuse problems due to the fear of them abusing prescription stimulants such as amphetamines, even though there is substantive evidence that treatment with amphetamines can improve both conditions (ADHD and CUD) ${ }^{4,5,9,10,11}$. In conclusion, amphet- 
amines have been found to improve both ADHD and CUD symptomatology primarily through increasing DA release from nerve terminals in the central nervous system, as well as increasing the release of NE and serotonin $^{4,9}$. However, their use in patients with CUD must be monitored closely due to their high potential for abuse.

\section{Acknowledgements}

The author would like to acknowledge Dr. Spring Farrell for her feedback on this paper and for how well she taught the material and theory on which this paper is based. The author is of the firm belief that good professors and teachers open the minds and expand the horizons of their students, bringing out the best in them.

\section{References}

1. American Psychiatric Association. Diagnostic and statistical manual of mental disorders (5th ed.). Washington, DC: American Psychiatric Association, 2013.

2. Brahmbhatt $\mathrm{K}$ et al. Diagnosis and Treatment of Attention Deficit Hyperactivity Disorder During Adolescence in the Primary Care Setting: A Concise Review. J Adolesc Health 2016;59(2):135-143.

3. Substance Abuse and Mental Health Services Administration (SAMHSA). "Treatment of Stimulant Use Disorders." 2020. $<$ https://store.samhsa.gov/sites/default/files/SAMHSA_Digital_ Download/PEP20-06-01-001_508.pdf> (30 November 2020).

4. Levin FR et al. Extended-release mixed amphetamine salts vs placebo for comorbid adult attention-deficit/hyperactivity disorder and cocaine use disorder: a randomized clinical trial. JAMA psychiatry 2015;72(6):593-602.

5. Wilens $\mathrm{T}$ et al. Misuse and Diversion of Stimulants Prescribed for ADHD: A Systematic Review of the Literature. J Am Acad Child Adolesc Psychiatry 2008;47(1): 21-31.

6. Heal D, Smith S, Gosden J, Nutt D. Amphetamine, past and present - a pharmacological and clinical perspective. J Psychopharmacol 2013;27(6):479-496.

7. DrugBank. "Amphetamine." 2020. <https://www.drugbank.ca/ drugs/DB00182> (25 November 2020).

8. Sharbaf Shoar N, Marwaha R, Molla M. Dextroamphetamine-amphetamine. In: StatPearls. Treasure Island, FL: StatPearls Publishing, 2020.

9. Mooney ME et al. Effects of oral methamphetamine on cocaine use: a randomized, double-blind, placebo-controlled trial. Drug Alcohol Depend 2009;101(1-2):34-41.

10. Levin FR, Choi CJ, Pavlicova M. How treatment improvement in ADHD and cocaine dependence are related to one another: a secondary analysis. Drug Alcohol Depend 2018;188:135-140.

11. Levin FR, Bisaga A, Raby W. Effects of major depressive disorder and attention-deficit/hyperactivity disorder on the outcome of treatment for cocaine dependence. J Subst Abuse Treat 2008;34(1):80-89.

12. Ontario College of Pharmacists. "Opioid Policy." Practice Policies and Guidelines. 2018. <https://www.ocpinfo.com/ regulations-standards/practice-policies-guidelines/opioid-policy/> (5 May 2021). 